\title{
Die rhetorischen Strategien von @realDonaldTrump und die Verweigerung diskursiver Argumentation
}

\author{
Gyburg Uhlmann
}

\section{Einleitung}

2018 wurde von der amerikanischen Öffentlichkeit und den Medien eine neue Entwicklung mit Aufmerksamkeit und Kritik verfolgt. Diese Entwicklung betrifft die Anzahl, Häufigkeit und Länge von Press Briefings im Weißen Haus, also von regelmäßigen Informationen und Fragestunden der Pressestelle mit den im Weißen Haus akkreditierten Journalist*innen, und von On-the-record-Pressekonferenzen mit Donald Trump (vgl. z. B. Breuninger 2018; Samuels 2018a). Seit Januar 2018 hatte die Pressestelle des Weißen Hauses unter der Leitung von Pressesprecherin Sarah Sanders die Anzahl der Press Briefings und ihre Dauer immer weiter reduziert. Stellte sie sich im Januar 2018 noch elfmal den Fragen der Reporter*innen, so waren es im Juni 2018 noch fünf, im Juli drei, im August fünf und im September sogar nur ein einziges Briefing. Auch die Dauer der Briefings wurde verkürzt, so dauerte die Fragestunde im September nur 18 min (Breuninger 2018; Winck und Shankar 2018).

Als Begründung für diese gegenüber anderen Administrationen veränderte Praxis führte Sarah Sanders an (Samuels 2018b), dass an die Stelle der klassischen Briefings eine Vielzahl anderer Gelegenheiten getreten sei, bei denen sich der Präsident selbst Fragen der Journalist*innen stelle: in kurzen Frage-AntwortSessions, bei Treffen mit ausländischen Staatsgästen, bei Kabinettssitzungen. In all diesen Situationen gebe es Gelegenheit, den Präsidenten selbst zu befragen. Hinzu kommt, dass Trump verglichen mit anderen Präsidenten seit Ronald Reagan am zweithäufigsten Interviews gegeben hat. Außerdem äußert er sich auf Twitter mit einer durchschnittlichen Anzahl von mehr als neun Tweets pro Tag (2018).

G. Uhlmann $(\triangle)$

Berlin, Deutschland

E-Mail: g.uhlmann@fu-berlin.de 
Das heißt also: Es gibt mehr, aber kürzere und für den Präsidenten kontrollierbarere Situationen, in denen Nachfragen von der Presse gestellt werden können. Das macht auch die Gemeinsamkeit der Entwicklungen in den Press Briefings mit dem Hauptkommunikationskanal, den Trump und damit auch seine Administration wählt, nämlich dem Kurznachrichtendienst Twitter, aus: Der Präsident bringt die Kommunikationswege soweit wie möglich unter seine Kontrolle und Regie. Während bei längeren Frage-Antwort-Gelegenheiten die Themen offener und eher den Journalist*innen anheimgestellt sind, ist es schwieriger und dem Anlass potenziell unangemessen, z. B. in der Anwesenheit von internationalen Staatsgästen oder vor Beginn von Kabinettssitzungen kleinteilige innenpolitische Fragen oder Kritik aufs Tapet zu bringen.

Der Unterschied, der entsteht, wenn das klassische Press Briefing durch die Pressesprecherin mehr oder weniger wegfällt oder doch stark eingeschränkt wird, ist aber noch ein anderer: Traditionell müssen Pressesprecher*innen auch harten Fragen gegenüber geradestehen und die Politik der Regierung erklären, begründen, rechtfertigen, in die richtigen Kontexte stellen usw. Die Presse kann die Regierungsarbeit hier freier als in der dem Amt des Regierungschefs gebührenden respektvollen Haltung diesem direkt gegenüber hinterfragen. Auch wenn Sanders betont, dass es doch immer besser und Zeichen größerer Transparenz sei, die Aussagen direkt vom Präsidenten zu hören, so hat es doch auch einen Transparenz behindernden Effekt, wenn es immer nur der Präsident selbst ist, dem - allerdings immer weniger - Fragen gestellt werden. Und auch wenn Sanders betont, sie wolle bei ihren Pressekonferenzen verhindern, dass Selbstdarstellungsexzesse (der Journalist*innen) überhandnähmen, hat gerade die Strategie, die Pressevertreter*innen nur noch direkt mit Präsident Trump zu konfrontieren, genau diesen Effekt, wie man exemplarisch in der Auseinandersetzung zwischen Trump und dem $C N N$-Journalisten Jim Acosta bei einer Pressekonferenz am 7. November 2018 sehen konnte, aufgrund derer Acosta zwischenzeitlich die Akkreditierung zum Weißen Haus entzogen worden war. Beide Akteure, sowohl der $C N N$-Reporter Acosta als auch Präsident Trump, nutzten die Pressekonferenz als Forum, um eine Botschaft zu vermitteln. Acosta hatte Trump nämlich auf eine Formulierung angesprochen, die dieser für eine große Karawane von Immigrant*innen gewählte hatte. Diese waren von Mittelamerika durch Mexiko bis an die US-amerikanische Grenze gezogen, um dort Asyl zu beantragen. Trump nannte diese Karawane eine ,invasion“: „,[Acosta]: I wanted to challenge you on one of the statements that you made in the tail end of the campaign in the midterms, that this - [...] caravan was an invasion." (Washington Times Staff 2018)

Acosta ließ auch nach einer ersten Antwort des Präsidenten nicht locker, woraufhin Trump versuchte, ihm das Mikrofon entziehen zu lassen. Auf Acostas Weigerung hin formulierte er mit Wut eine Generalkritik an Acosta, dem ,Fake News-Sender' $C N N$ und dessen grundsätzlichen Unglaubwürdigkeit.

Man sieht: Selbstdarstellung und Bloßstellung des anderen sind der primäre Motor der Auseinandersetzung. Wo aber bleibt dann angesichts dieser von Trump gewählten Medienstrategien noch Raum für den Austausch von Argumenten, von Für und Wider, von Sachargument und Gegenargument? Wo ist noch Raum für das 
längere Argument und langfristige Perspektiven? Wo können frei Fragen gestellt und mit der notwendigen Ruhe beantwortet werden?

Die Wahl der Medien der Kommunikation und Selbstdarstellung haben, wie hieraus deutlich wird, selbst eine politische Qualität. Das bedeutet freilich nicht, dass die Kommunizierenden den Zwängen der Medien einfach unterliegen, sondern vielmehr dass die Wahl der Medien und die Techniken der Kommunikation mit der politischen Agenda der Akteure interagieren, und dass die kommunikativen Strategien auch das jeweilige Medium formen und verändern können.

In diesem Beitrag wird die Kommunikation Donald Trumps über den Kurznachrichtendienst Twitter unter genau diesem Gesichtspunkt untersucht: Was geschieht, wenn ein amerikanischer Präsident seine Politik vor allem über einen die Länge der Äußerungen stark beschränkenden Nachrichtendienst vermittelt und praktiziert? Dafür werde ich in zwei Schritten vorgehen: Ich beginne mit einem Überblick über die Twitter-Strategien und mit Fallstudien, wie die Kommunikation und die Reaktionen darauf ablaufen. Im zweiten Schritt hebe ich die Frage auf eine höhere Ebene und untersuche die an diesen Strategien beteiligten Faktoren (wie Medium und Argumentationsmethode usw.) kritisch dadurch, dass ich sie mit Diskussionen aus der antiken Rhetoriktheorie in Verbindung bringe, die genau solche Fragen zum Thema haben: Was zeichnet die Qualität einer politischen Kommunikation aus? Welche Faktoren sind dafür entscheidend und was bedeutet die Wahl bestimmter Medien und Techniken für den Gehalt der so vermittelten Politik?

\section{Trump und Twitter}

Warum also nutzt Trump Twitter und wie tut er das? Es gibt verschiedene Merkmale oder Auffälligkeiten der rhetorischen Strategien, die Donald Trump in seiner Twitter-Kommunikation verwendet. Um diese einordnen zu können, müssen wir zunächst bestimmen, was unter ,rhetorischen Strategien` zu verstehen ist. Das ist insbesondere deswegen wichtig, weil Trumps Twitter-Stil häufig als spontan, ungeplant, affektbestimmt, von Wut getrieben, einfach dumm (Urback 2017) oder gar mental instabil ${ }^{1}$ beschrieben wird, sodass der Gedanke aufkommen könnte, dass wir es gar nicht mit einer Technik im eigentlichen Sinn zu tun haben. Rhetorik umfasst nach dem hier zugrunde gelegten Begriff alle Formen der strategischen Kommunikation und beschränkt sich demnach weder auf bestimmte Medien noch auf die Anwendung bestimmter traditioneller Techniken.

Meine These ist, dass Trumps Twitter-Kommunikation durchgehend strategisch und somit rhetorisch ist und die Möglichkeiten des Kurznachrichtendienstes nutzt und sogar selbst weiterentwickelt, um sachlichen Diskussionen auszuweichen und rationale Argumentationen zu meiden. Meine Analysen konzentrieren sich dabei

\footnotetext{
${ }^{1}$ Dagegen argumentiert Stöber 2011.
} 
nicht wie oftmals üblich auf die Verwendung von Stilfiguren, den formalen Aufbau einer Rede, Humoristisches oder Spannungsbögen, sondern betrachten für die Bestimmung der strategischen Qualität der Rhetorik Trumps besser geeignete Indikatoren wie die Nutzung von einprägsamen Formeln anstelle von genauen Sachanalysen oder das Etikettieren von Gegner*innen als Ersatz für eine Auseinandersetzung mit ihren Argumenten und Positionen oder auch die Nutzung bestimmter Medien und das Ausblenden von Tatsachen oder das Ausweichen auf Nebenschauplätze der Auseinandersetzung. Auf diese Weise wird deutlich, dass Twitter ein idealer Untersuchungsgegenstand ist, weil hier die prominentesten und zugleich problematischsten Seiten von Trumps Politik und seiner Rhetorik Wirkung entfalten: nämlich Strategien, die anti-diskursiv, anti-rational, dekontextualisierend und manipulativ emotionalisierend sind und die an die Stelle begründeter Argumentationen eindrucksvolle Bilder, Merksätze, einprägsame Wendungen und Beleidigungen setzen. Neben solchen Techniken des Dekontextualisierens spielen dabei auch Strategien der Wahrheitsbehauptung oder das Anprangern von Lügen anderer eine wichtige Rolle.

Ich beginne mit einem Detail, das auf den ersten Blick wie ein rein formaler und stilistischer Aspekt erscheinen kann, das sich jedoch im Laufe genauerer Analysen als Symptom allgemeiner und kommunikationsstrategischer Methoden erweisen wird:

1. Merkmal: Emotionale Lautstärke durch Formatierung, Wiederholung und Abschluss von Aussagen bzw. Tweets mit emotionalen Ausdrücken anstelle von Begründungen

Trumps Twitter-Stil wird oft als spontan und emotional beschrieben. Diese Einschätzung hat unterschiedliche Gründe. Dazu gehört z. B. Trumps Neigung, die Korrespondenz mit ausländischen Regierungschefs gerade in Zeiten der Konfrontation über Twitter (und vermutlich ohne vorherige Beratung mit seinem Team und seiner Kommunikationsabteilung) und jenseits der Regeln der diplomatischen Etikette zu führen. Berühmt und viel kritisiert wurde folgender Tweet vom 2. Januar 2018, den Trump im Zuge des Atomkonflikts mit dem nordkoreanischen Staatschef Kim Jong Un verfasste:

\footnotetext{
„North Korean Leader Kim Jong Un just stated that the ,Nuclear Button is on his desk at all times'. Will someone from his depleted and food starved regime please inform him that I too have a Nuclear Button, but it is a much bigger \& more powerful one than his, and my Button works!“ (2. Januar 2018, @ realDonaldTrump)
}

$\mathrm{Zu}$ diesem Eindruck trägt aber auch ganz wesentlich die oft emotionale oder emotionalisierende Sprache der Tweets bei, ${ }^{2}$ die häufig an die Stelle einer näheren Beschreibung oder Analyse eines Sachverhalts ein emotionales Adjektiv oder

\footnotetext{
${ }^{2}$ Vgl. die Analysen von Brady et al. 2017.
} 
Substantiv stellt oder aber auch durch die Verwendung von Großbuchstaben die Meinungsäußerung, lautstark' unterstreicht. Trump nennt Handlungen, Situationen, Aussagen „,verrückt“ (,,crazy“) oder verlogen oder stellt fest, dass etwas eine „Katastrophe“ (,disaster“) ist. Das von Trump am häufigsten gewählte Wort für die Charakterisierung und Analyse eines Sachverhalts aber ist „sad“. Oftmals charakterisiert Trump das Verhalten der Demokraten als ,traurig', ebenso aber auch das Verhalten der Presse:

„Democrats are trying to bail out insurance companies from disastrous \#ObamaCare, and Puerto Rico with your tax dollars. Sad!“ (12:06 - 27. April 2017, @ realDonaldTrump)

Gerade zu Beginn seiner Präsidentschaft, als Trump die ersten Schritte unternahm, um das Krankenversicherungsprogramm ,ObamaCare" wieder abzuschaffen, nannte er dieses Programm häufig eine „Katastrophe“.3 Die Wiederholung dieser Auffassung ersetzte in Trumps Rhetorik die kleinteilige Auseinandersetzung mit Vor- und Nachteilen des von Präsident Obama eingeführten Systems und vor allem auch das Offenlegen tragfähiger Alternativen. Etwas Ähnliches gilt für die Geschehnisse rund um die Entlassung des FBI-Chefs James Comey, auf die wir gleich noch zurückkommen werden. Auch hier wendet Trump die Auseinandersetzung mit den Demokraten ins Emotionale: „Dems have been complaining for months \& months about Dir. Comey. Now that he has been fired they PRETEND to be aggrieved. Phony hypocrites!“(10. Mai 2017, @ realDonaldTrump)

Trump verwendet diese Emotionalisierung aber auch im Bereich des Show Business und Entertainments ${ }^{4}$ oder des Sports, von denen, wie das nächste Beispiel zeigt, in seiner Kommunikation allerdings die Grenzen zum Politischen und überhaupt zwischen unterschiedlichen Diskursen und gesellschaftlichen Sphären verschwimmen: ,While not at all presidential I must point out that the Sloppy Michael Moore Show on Broadway was a TOTAL BOMB and was forced to close. Sad!“‘ (28. Oktober 2017, @ realDonaldTrump) ${ }^{5}$

Dadurch, dass die Charakterisierung, sad“ häufig an das Ende eines Tweets gestellt wird, fasst sie die gesamte Aussage und Situation zusammen und verknüpft die von Trump ausgesprochene und dadurch evozierte Emotion eng mit

\footnotetext{
${ }^{3}$ Diese Meinung wird auch in anderen Medien vermittelt, wie z. B. bei einer Monday address Donald Trumps vor der National Governors Association am 27.02.2017: „I see it happening with Obamacare. People hate it, but now they see that the end is coming and they say, ,Oh, maybe we love it. " There's nothing to love - it's a disaster, folks, OK? So you have to remember that." (Smith 2017)

${ }^{4}$ Z. B. „The @TheView @ABC, once great when headed by @BarbaraJWalters, is now in total freefall. Whoopi Goldberg is terrible. Very sad!“‘(10:06 PM - 7. Januar 2016, @ realDonaldTrump)

${ }^{5}$ Ein weiteres Beispiel: „NBC NEWS is wrong again! They cite ,sources“ which are constantly wrong. Problem is, like so many others, the sources probably don't exist, they are fabricated, fiction! NBC, my former home with the Apprentice, is now as bad as Fake News CNN. Sad!“ (03:45 - 4. Mai 2018, @ realDonaldTrump)
} 
dieser. Eine solche Strategie kann insbesondere dann Wirkung zeigen, wenn sie in ähnlichen Konstellationen wiederholt wird. Das Verhalten der Medien gegenüber Trump, seinem Team, seinen Entscheidungen usw. ist eben immer , sad', die politischen Reaktionen der Demokraten ebenso.

In politischen Kontexten entbehren solche Behauptungen oftmals eines konkreten Anlasses (s. u.) oder lassen sich in dieser Form und Allgemeinheit nicht aufrecht halten. Die Medien führen dazu lange Listen von Fällen, in denen Trump pauschalisierende oder nicht den Tatsachen entsprechende Aussagen gemacht hat. Die emotionale Aufladung allerdings kann durch solche Kritiken oder Widerlegungen oftmals nicht oder nur schwer rückgängig gemacht werden, besonders dann, wenn Trump die Kritik einfach ignoriert. Das trifft auf Trumps Zielpublikum $\mathrm{zu}$, dessen elitenfeindliche Haltung Trump durch das Erzeugen und Präsentieren derartiger Emotionen aufgreift und verstärkt. Doch auch bei anderen Leser*innen verfestigt sich der Eindruck einer emotionalen Debatte und davon, dass die von Trump emotional forcierte Lagerbildung und Spaltung der Gesellschaft immer mehr um sich greift. Diese Atmosphäre kann auch auf der Seite der Attackierten $\mathrm{zu}$ emotionalen Gegenreaktionen führen. Beispiele dafür gibt es in den Repliken des professionellen Journalismus auf Trumps unwahre oder einseitig verzerrende Aussagen oder in den Interviews von Trumps Berater*innen bei $C N N$ oder NBC (usw.) zuhauf. Doch auch der professionelle Mainstream-Journalismus ist keineswegs frei von selbst initiierten Emotionalisierungen, die von der Sachdiskussion - zumindest potenziell - wegführen können. So ist es auch im deutschen Journalismus gang und gäbe, politische Problemlagen anhand eines Einzelfalls darzustellen. Das macht die Sachfrage konkreter und anschaulicher, trägt aber auch die Schwierigkeit in sich, dass die Zuhörenden oder Zuschauenden dazu angeleitet oder verleitet werden, sich so sehr mit dem vorgestellten Einzelschicksal zu identifizieren und mitzufühlen, dass das Abwägen der Argumente Pro und Contra durch diesen Blick auf z. B. das individuelle Leid des als Beispiel präsentierten Menschen behindert wird. Es bleibt eine hohe Kunst, dafür zu sorgen, dass das Streben nach Anschaulichkeit und Konkretheit nicht unmerklich in einen Betroffenheitsjournalismus übergeht, der das angestrebte Ziel, umfassend zu informieren und den Rezipient*innen eine eigenständige und mündige Meinungsbildung zu ermöglichen, aus den Augen verliert. Auch ein solcher Journalismus liefe dann Gefahr, manipulative rhetorische Techniken zu verwenden. ${ }^{6}$

\section{Merkmal: Dekontextualisierung}

Trump mischt auf seinem offiziellen Twitter-Account @ realDonaldTrump verschiedenste Themen bunt durcheinander. Dort formuliert er ebenso Nachrichten an Staatschefs anderer Nationen wie Reaktionen auf Ergebnisse im Football oder kommentiert Fernsehsendungen verschiedenster Genres. Dabei neigt Trump dazu,

\footnotetext{
${ }^{6}$ Differenzierte Beschreibungen zu manipulativen Strategien und dem Einsetzen von plastischen Vorstellungsbildern in der Rhetorik lege ich in Uhlmann 2019 vor.
} 
die einzelnen Beiträge nicht thematisch einzuführen und zu verorten. Er schreibt nicht oder nicht immer, worauf sich der Kommentar oder die Replik, die er formuliert, bezieht. Ein Beispiel: Am 7. Januar 2019 postete Trump zwei wütende Tweets, in denen er die Medien insgesamt der Fake News bezichtigt und seinen Vorwurf, die Medien seien der Feind des amerikanischen Volkes (,enemy of the American people“) wiederholt. ${ }^{7}$ Exemplarisch sind die Tweets vom 7. Januar um 17:09 und 17:31 nachmittags, weil in diesen zunächst unklar blieb, was der Anlass für die Vorwürfe gegenüber den Medien war. Wahrscheinlich gibt ein $1 \frac{1 / 2}{h}$ später geschriebener Tweet Trumps einen Hinweis zur Lösung des Rätsels. In diesem beklagte sich Trump nämlich darüber, dass die New York Times über seine Absichten beim Rückzug der amerikanischen Truppen aus Syrien falsch berichtet habe. Die Tweets folgten jedoch nicht direkt aufeinander. Dazwischen wurde noch ein Tweet bezüglich des Shutdowns der Bundesverwaltung veröffentlicht, den es wegen eines Streits um den Haushalt Anfang 2019 gab. Der direkte Zusammenhang zwischen den Beschuldigungen an die Medien kann also nur indirekt und wahrscheinlich erschlossen werden.

Das Mischen von Nachrichten mit innenpolitischem, sportlichem oder gar persönlichem Inhalt und Nachrichten, die sich (zumindest in der direkten Anrede) an Staats- und Regierungschefs anderer Nationen wenden, ist ein weiterer Aspekt solcher Dekontextualisierungen. Es handelt sich hier jedoch eher um eine Dekontextualisierung vor dem Hintergrund etablierter Regeln in der internationalen politischen Kommunikation, mit denen Trump explizit bricht. Twitter-Nachrichten, die direkt von einem Präsidenten veröffentlicht werden, müssen hier schon von vorneherein als eine unübliche Kommunikationspraxis gelten, die auch zum Teil massive Kritik hervorrief.

Ein Beispiel dafür sind die Tweets Donald Trumps an die Türkei bzw. die Konfliktparteien türkische Regierung und kurdische Truppen, mit denen die USA in der Bekämpfung der Terrormiliz ,Islamischer Staat' zusammengearbeitet hatte:

„Starting the long overdue pullout from Syria while hitting the little remaining ISIS territorial caliphate hard, and from many directions. Will attack again from existing nearby base if it reforms. Will devastate Turkey economically if they hit Kurds. Create 20 mile safe zone [...].“(14:53 - 13. Januar 2019, @ realDonaldTrump)

Dieser erste Tweet wurde fortgesetzt durch:

„[...] Likewise, do not want the Kurds to provoke Turkey. Russia, Iran and Syria have been the biggest beneficiaries of the long term U.S. policy of destroying ISIS in Syria natural enemies. We also benefit but it is now time to bring our troops back home. Stop the ENDLESS WARS!“ (15:02 - 13. Januar 2019, @ realDonaldTrump)

Der türkische Außenminister Mevlut Cavusoglu beschrieb den Tweet als eine „domestic policy message“ und kritisierte, dass Trump sich über Twitter und

\footnotetext{
${ }^{7}$ Trump hatte diese Formulierung „enemy of the American people“ im Jahr 201816 mal und im Januar 2019 weitere vier Male auf Twitter benutzt (vgl. Davis 2018).
} 
nicht in einer persönlichen Ansprache an seine Kollegen in der Türkei gewendet habe (,Strategic alliances should not be discussed over Twitter or social media“). Der Eindruck, dass die politische Aussage deplatziert ist, wurde von Trump selbst sogleich dadurch weiter verschärft, dass er sich in dem unmittelbar folgenden Tweet mit einem bösen Seitenhieb auf den Amazon-Chef Jeff Bezos im Zusammenhang mit dessen Trennung von seiner Frau zu Wort meldete:

„So sorry to hear the news about Jeff Bozo being taken down by a competitor whose reporting, I understand, is far more accurate than the reporting in his lobbyist newspaper, the Amazon Washington Post. Hopefully the paper will soon be placed in better \& more responsible hands.“(17:45 - 13. Januar 2019, @ realDonaldTrump)

Der Konflikt mit Bezos hat für Trump freilich (wirtschafts- und medien-)politischen Charakter, gleichwohl bezieht sich der Tweet unmittelbar auf eine Angelegenheit, die sonst eher in der Klatschpresse verhandelt wird. Denn Trump spielt in dem Tweet auf einen Artikel des National Enquirer an, der behauptet, Beweise für eine Affäre Bezos' mit der Moderatorin Lauren Sanchez vorlegen zu können. Durch die Verunglimpfung des Namens Bezos zu ,Bozo‘, einer bekannten amerikanischen Clown- und Kinderbuchfigur, wird die Invektive verstärkt und auf die Ebene von Illustrierten und Unterhaltungsmedien versetzt.

Der an die Konfliktparteien im Syrienkonflikt adressierte (und freilich zugleich die heimischen Wähler ansprechende) Tweet erscheint damit noch einmal umso mehr dekontextualisiert. Eine Vermischung unterschiedlichster Kommunikationsebenen scheint Trump nicht nur in Kauf zu nehmen, sondern ostentativ zum Prinzip seiner Äußerungen als öffentlicher Person zu machen. Ryan Skinnell argumentiert in diesem Zusammenhang, dass diese „transparency“ seiner gesamten Persönlichkeit (und Emotionalität) Teil einer Strategie ist, die die Authentizität des Redners und Kommunikators Trump beweisen möchte (Skinnell 2018b). Sie ist also eine rhetorische Strategie und zugleich damit eine, die politisch große Wirkungen zeigt und die Trumps Politikstil und -agenda prägt.

\section{Merkmal: Universeller Einsatz von Beleidigungen}

Invektive und Beleidigung sind auch in der öffentlichen Wahrnehmung die hervorstechendsten Elemente der Kommunikationspraktiken Trumps, und zwar auf allen kommunikativen Ebenen. Trump beleidigt politische Akteure in der Innenpolitik ${ }^{8}$ ebenso wie in der Außenpolitik, ${ }^{9}$ genauso aber auch andere Persönlichkeiten, die im Fokus der US-amerikanischen Öffentlichkeit stehen oder mit

\footnotetext{
${ }^{8}$ Herausragende Beispiele sind die Attacken auf Hillary Clinton, Chuck Chumer, James Comey, seit Ende 2018 auch Elizabeth Warren.

${ }^{9}$ Berühmtes Beispiel ist ein Tweet mit einer angedeuteten Beleidigung Kim Jong-uns: „Why would Kim Jong-un insult me by calling me old, when I would NEVER call him short and fat? Oh well, I try so hard to be his friend - and maybe someday that will happen!", die Trump als Reaktion auf einen persönlichen Angriff Kims am 12. November 2017 um 1:48
} 
denen Trump persönliche Konflikte oder Feindschaften pflegt. Doch die Analyse würde zu kurz greifen, wollte man dieses Faktum allein als Marotte oder als Zeichen schlechter Manieren abtun. Denn dann verfehlte man den strategischen Charakter hinter den Äußerungen. Dass es sich um Strategien handelt, ist allein schon deswegen plausibel, weil Trump beleidigende Nicknames und andere Formen der Beleidigungen konstant und als wiederkehrende Elemente einsetzt, vor allem aber, weil diese Beleidigungen in kommunikativen Situationen eingesetzt werden, die eigentlich eine sachliche Auseinandersetzung erforderten. Aus den Kontexten und der Art ihrer Verwendung kann man schließen, dass die Beleidigungen bestimmte Funktionen in den kommunikativen Strategien Trumps besitzen. Dabei spielt es noch nicht einmal die entscheidende Rolle, ob diese strategischen Techniken genauso intendiert sind oder ob es sich nicht vielleicht doch um psychologisch zu erklärende, vielleicht gar krankhaft narzisstische, Verhaltensweisen (vgl. Milbank 2017) handelt. Viel wichtiger ist ihre Wirkung. Denn die rhetorische Qualität einer sprachlichen Äußerung hängt ganz wesentlich davon ab, wie die Rede ankommt und wie sie zur Überzeugung der Rezipient*innen beiträgt.

Beispiele für strategische Beleidigungen gibt es wie gesagt zuhauf. An anderer Stelle habe ich ausführlicher die Verwendung beleidigender Nicknames betrachtet und diese als ,demagogische Epitheta“ charakterisiert (Uhlmann 2019) um darauf hinzuweisen, dass es sich nicht um bloße Verunglimpfungen und Unbeherrschtheiten handelt, sondern dass die Funktion dieser Beiworte darin besteht, ein festes Bild von der jeweiligen Person zu etablieren, in dem Bezeichnungen wie ,lügnerisch', ,aalglatt', ,betrügerisch ' oder ,verrückt' suggerieren, es handele sich dabei um permanente Eigenschaften dieser Personen, die sie so sehr für alles diskreditieren, dass eine weitere Auseinandersetzung mit deren Argumenten als von vorneherein nicht erforderlich erscheint.

In Situationen und auf einem Terrain, das für Präsident Trump ein Minenfeld darstellt, nehmen die Attacken oftmals nicht nur $\mathrm{zu}$, sondern in den Tweets werden auch unterschiedliche Beleidigungen und Kampfschauplätze miteinander vermischt. Aussagekräftige Beispiele für diese rhetorische Strategie finden sich immer wieder in Äußerungen, die sich auf die Russlandaffäre beziehen, also auf die Untersuchungen darüber, ob Trump und sein Wahlkampfteam 2016 mit der russischen Regierung in Kontakt getreten sind, um von russischen Hacker*innen Unterstützung durch Manipulationen in den sozialen Netzwerken zu erhalten. Trump hatte den damaligen FBI-Chef James Comey am 9. Mai 2017 entlassen, woraufhin Vorwürfe laut wurden, die Entlassung stünde im Zusammenhang mit den von Comey initiierten Ermittlungen des FBI gegen Trump. Zu Trumps Repliken auf diese Vorwürfe hatte seitdem immer wieder gehört, Comey Nachlässigkeit

(@ realDonaldTrump) während seiner Asienreise twitterte. Trump reagierte damit auf eine Äußerung Jong-uns, der im Rahmen einer Fernsehansprache am 22. September 2017 gesagt hatte: „I will surely and definitely tame the mentally deranged US dotard with fire“, wobei „dotard“ im Sinne von ,alter Tattergreis“" gemeint ist. 
und Parteilichkeit vorzuwerfen, weil er in der E-Mail-Affäre um Hillary Clinton angeblich nicht in demselben Maße Engagement und Hartnäckigkeit gezeigt habe.

Im Januar 2019 während des Hauhaltsstreits, der zu einem beispiellos langen Shutdown der amerikanischen Bundesverwaltung geführt hatte und Trump immer weiter unter Druck setzte, seine Forderungen nach der Finanzierung einer Mauer an der Südgrenze der USA zu Mexiko aufzugeben, verknüpfte Trump nun diese Konflikte und kam noch einmal auf die Comey-Affäre zurück:

\begin{abstract}
„Wow, just learned in the Failing New York Times that the corrupt former leaders of the FBI, almost all fired or forced to leave the agency for some very bad reasons, opened up an investigation on me, for no reason \& with no proof, after I fired Lyin' James Comey, a total sleaze!““(04:05 - 12. Januar 2019, @ realDonaldTrump)
\end{abstract}

Und wenige Minuten später schreibt er:

„[...] Funny thing about James Comey. Everybody wanted him fired, Republican and Democrat alike. After the rigged \& botched Crooked Hillary investigation, where she was interviewed on July 4th Weekend, not recorded or sworn in, and where she said she didn't know anything (a lie), [...].“(04:18 - 12. Januar 2019, @ realDonaldTrump)

Trump nennt in einer Fortsetzung dieses Tweets Comey zudem noch einen „Crooked Cop“, also einen „,verlogenen Polizisten“, in Anspielung auf Trumps ,Standard-Beleidigung “ „Crooked Hillary“ für Hillary Clinton. ${ }^{10}$

Durch diese Beleidigungen polarisiert Trump und bildet oder verstärkt die Wirkung seiner populistischen Rhetorik, die klare Gegner und Fronten identifiziert. Die Beleidigten werden als „Feinde des amerikanischen Volkes“ (eine Beschreibung, die Trump allerdings nur auf die Medien - mit Ausnahme des Senders Fox $^{11}$ - bezogen ausgesprochen hat) diskreditiert und alles, was sie sagen oder tun, damit als etwas charakterisiert, über das es gar nicht lohnt, weitere Gedanken oder gar argumentative Auseinandersetzungen zu verwenden.

\title{
4. Merkmal: Selbstzitate von Beleidigungen und (oft invektiven) Narrativen
}

Donald Trump reflektiert selbst auf seine Praxis, Kontrahent*innen mit herabsetzenden Namen zu belegen, und zwar in einer Weise, die den strategischen Charakter und die angestrebten Effekte deutlich ins Auge rückt. Dies ist z. B. in der Auseinandersetzung mit seinem Konkurrenten um die Nominierung zum Präsidentschaftskandidaten 2016, Ted Cruz, der Fall, den Trump als „Lyin“ Ted“ titulierte. Am 6. April 2016 bei einem Wahlkampfauftritt in Bethpage, New York,

\footnotetext{
${ }^{10}$ In dem Tweet auf @ realDonaldTrump um 04:33 - 12. Januar 2019.

${ }^{11}$ Aufschlussreich für Trumps Sortierung der Medienlandschaft der USA ist eine Pressekonferenz vom 16. Februar 2017, in der er die Ehrlichkeit von Fox (News) im Unterschied zu den Sendern, die ihm mit Hass und Verfolgung begegneten, wie $C N N$ hervorhebt: https://youtu.be/g6CATPB0o9o?t=2685 (08.01.2019).
} 
bekannte er sich dazu, dass er die Formel „Lyin“ Ted“, die seine Anhänger*innen lautstark skandierten, erfunden habe, um die Notwendigkeit zu betonen, diese Formel richtig zu schreiben (vgl. Uhlmann 2019). Diese Praxis verstärkte Trump während seiner Präsidentschaft noch weiter. So attackierte er die demokratische Senatorin Elizabeth Warren, indem er sie „Pocahontas“ nannte. Er benutzte diesen Namen aber nicht als Beinamen, vielmehr ersetzte der Name Warrens eigentlichen Namen ganz. Warren rückte seit dem Herbst 2018 zunehmend in den Fokus von Trumps Beleidigungen und Narrativen, auf die sich Trump dann mitunter auch selbst zurückbezieht, wie z. B. in dem folgenden Tweet, der ein bei Instagram veröffentlichtes Video Warrens, gesendet aus ihrem privaten Haus, kommentiert:

„If Elizabeth Warren, often referred to by me as Pocahontas, did this commercial from Bighorn or Wounded Knee instead of her kitchen, with her husband dressed in full Indian garb, it would have been a smash!“ (Dann zitiert Trump ein Instagram-Video von Elizabeth Warren vom 31. Januar 2018 auf @ realDonaldTrump, 18:52 - 13. Januar 2019.)

Pocahontas (ca. 1595-1617) war eine Tochter des native american chief Powhatan, von der der englische Abenteurer John Smith berichtet, sie habe ihm einmal das Leben gerettet. Ursprünglich geht diese Benennung durch Trump auf eine Episode aus dem Jahr 2012 zurück. Damals versuchte Warren, mit dem Verweis auf ihre Herkunft native americans für ihren Wahlkampf um einen Sitz im Senat zu mobilisieren. Trump scheint ihr in diesem Zusammenhang vorgeworfen zu haben, die Wähler mit falschen oder doch nicht beweisbaren Fakten in die Irre zu führen. ${ }^{12}$ Denn Warren legte damals für ihre Behauptungen über ihre Abstammung keine Beweise vor. Seit 2018 gibt es diese Beweise für eine sehr entfernte Verbundenheit nun in der Form eines Gentests, den Warren durchführen ließ. In der zweiten Hälfte des Jahres 2018 passte Trump seine Strategien daher an die neue Situation an. Seit Warren mit Stolz die Belege für ihre Abstammung von native americans präsentiert, versucht Trump nun immer noch, Warren mit dem Namen zu degradieren und ihren Stolz auf ihre Herkunft als Koketterie und wegen der sehr entfernten Verwandtschaft mit native americans lächerlich $\mathrm{zu}$ machen. In jedem Fall aber arbeitet Trump zunehmend daran, seine politische Konkurrentin auf dieses politisch eigentlich unbedeutende Faktum und ihren Umgang damit zu reduzieren.

Trump entfaltet seinen oft genutzten Vergleich in unserem Text selbst zu einem Bild mit weiteren anschaulichen Details. Es liegt nahe, dass diese ausführliche Adressierung mit Warrens am 31. Dezember 2018 bzw. 1. Januar 2019 veröffentlichtem Entschluss zusammenhängt, als Präsidentschaftskandidatin gegen Trump antreten zu wollen. Der Anlass für die Zunahme an Engagement vonseiten Trumps in dieser Sache ist also transparent. Es ist aber vor allem der Effekt dieser

\footnotetext{
${ }^{12}$ Vgl. Franke-Ruta 2012 und „Thank you to the Cherokee Nation for revealing that Elizabeth Warren, sometimes referred to as Pocahontas, is a complete and total Fraud!“" (05:24 - 16.10.2018, @ realDonaldTrump). Trump bezeichnete die Wahlwerbung Warrens mit ihrer Herkunft sogar als rassistisch (Tweet vom 11.06.2016, 16:28).
} 
Strategie, der interessant ist. Je mehr Trump über die Herkunftsfrage spricht und je anschaulicher er ein Bild von Elizabeth Warren als ,Indianer-Squaw zeichnet, desto mehr steht diese Vorstellung dem Publikum dieser Auseinandersetzung auch dann vor Augen, wenn ganz andere und politisch tatsächlich relevante Sachverhalte verhandelt werden. Dabei blendet Trump die positive Besetzung der Figur Pocahontas aus. Denn auch wenn diese eine Identifikationsfigur ist, als Senatorin oder gar Präsidentin kann sie sich (etwa in der Form, wie die Geschichte in dem gleichnamigen populären Walt Disney-Film präsentiert wird) wohl kaum jemand vorstellen - und schon gar nicht jemanden, der nur vorgibt, dass in ihren Adern ,Indianerblut' fließe, ohne eine wirkliche Nähe zu dieser Minderheit zu besitzen. Zudem wurde schon verschiedentlich festgestellt und analysiert, dass Trump unbeirrt auch Gruppen attackiert, die mehrheitlich zu seinen Anhänger*innen zählen wie z. B. viele Kriegsveteranen, die Trump am 1. August 2016 vor den Kopf stieß, als er die Eltern eines im Irak gefallenen Soldaten wegen ihrer Kritik an seiner Politik beleidigte (vgl. Haberman und Oppel 2016). ${ }^{13}$ Der Name Pocahontas macht Elizabeth Warren politisch wenn nicht zu einer Witzfigur, so doch zu einer Gesprächspartnerin, die man nicht ernst nehmen kann. Die Strategie ist eben deshalb, weil sie die Gegnerin mittels eines Vorstellungsbildes auszuschalten versucht, perfide, anti-diskursiv und ein Ablenkungsmanöver von (in Zukunft zu führenden) politischen Diskussionen.

Mein letztes Beispiel nimmt die am Anfang referierte Situation auf: Am 22. Januar 2019 unternahm Donald Trump eine Attacke auf die Presse, mit der er erklären wollte, warum seine Pressesprecherin Sarah Sanders immer seltener Pressekonferenzen abhält: ${ }^{14}$

„The reason Sarah Sanders does not go to the ,podium“ much anymore is that the press covers her so rudely \& inaccurately, in particular certain members of the press. I told her not to bother, the word gets out anyway! Most will never cover us fairly \& hence, the term, Fake News!“‘ (7:28 - 22. Januar 2019, @ realDonaldTrump)

Die Erklärung bietet gleichzeitig erneut eine Begründung für einen der von Trump am häufigsten gebrauchten Ausdrücke, den Ausdruck ,Fake News“, der sich sowohl auf die einzelnen Berichterstattungen der großen Tageszeitungen und Fernsehanstalten wie New York Times, Washington Post oder CNN und ABC bezieht, als auch eine Charakterisierung für diese Zeitungen, Sender und ihre Journalist*innen selbst zu sein beansprucht. Nicht nur das, was sie schreiben und senden, seien Falschnachrichten, sondern die Reporter*innen und die Zeitung oder der Fernsehsender seien selbst Fake News. Damit wird die Taktik der Identifizierung von Gegner*innen mit bestimmten negativen (vermeintlich prädizierbaren) Eigenschaften auf die Spitze getrieben. Wenn Trump Reporter*innen immer wieder vorhält: „You are Fake News“ und wenn er - vor allem seinen Anhänger*innen wiederholt vorträgt, dass die Mainstreammedien grundsätzlich und in allem, was

\footnotetext{
${ }^{13}$ Vgl. zu diesen Attacken auf die eigenen Wählergruppen die Analysen von Skinnell 2018b.

${ }^{14}$ Vgl. dazu auch McArdle 2019.
} 
sie über Trump und seine Regierung sagen, nicht vertrauenswürdig sind, sondern habituell lügen und verzerren, dann wird die Dauereigenschaft, die Trump den Medien beleidigend zuordnet, sogar selbst zum Subjekt.

Die Idee, dass das Faktum, dass demokratische Mechanismen, zu denen auch traditionelle Formen der Informationspolitik und der Transparenz der Regierung wie die Press Briefings der Pressesprecherin gehören, außer Kraft gesetzt werden, selbst nur eine Reaktion auf das Fehlverhalten der Gegner darstelle, ist ein Tenor dieser neuartigen Form der Pressearbeit seit der Inauguration Trumps. Dies rückte zum ersten Mal in den Fokus der Öffentlichkeit, als die Trump-Beraterin Kellyanne Conway am 23. Januar 2017 in einem Interview mit dem Journalisten Chuck Todd in der Sendung „Meet the Press“ mit einer ähnlichen Erklärung für die Pressemitteilungen im Zusammenhang mit der Inaugurationsfeier am 22. Januar 2017 Kritik auf sich zog (Guterman 2017). Sie bezeichnete nämlich die Strategie der Kommunikationsabteilung des Weißen Hauses mit der Formel „We put out alternative facts." Mit diesen alternativen Fakten solle die feindselige und fehlerhafte Berichterstattung der großen Medienanstalten korrigiert und mit einem Gegengewicht versehen werden.

Das, was Trump und Conway tun, gehört zu der Elitenkritik Trumps, die ein wesentliches Moment seiner und generell populistischer Strategien ist. Die politik- und sozialwissenschaftliche Forschung zum Populismus hat diese Zusammenhänge bereits von vielen Seiten her aufgearbeitet und mit der Etablierung eines Überlegenheitsbewusstseins der jeweils adressierten Gruppe - „des Volkes“ und Abwertung anderer ausgegrenzter Gruppen in Verbindung gebracht (Müller 2016). Das sind zentrale Momente des Phänomens. Daneben stehen aber auch die rhetorischen, d. h. kommunikationsstrategischen Aspekte. Bei deren Analyse richten wir den Blick mehr auf die Strategien der Überzeugung und Vermittlung einer bestimmten Botschaft oder die Durchsetzung einer bestimmten Position mit sprachlichen und argumentativen Mitteln. Dabei wird deutlich, dass Trumps Narrative zu einer Verschiebung traditioneller Instrumente im politischen Diskurs führen. Sein Erfolg und seine Position ermöglichen es ihm, die klassischen (und sachlich berechtigten) Anforderungen, sich mit den Argumenten der Gegner auseinanderzusetzen, andere Positionen überhaupt erst einmal zur Kenntnis zu nehmen und sie richtig wiederzugeben, zu ignorieren. Wenn an ihre Stelle verzerrende und Nebensächliches ins Zentrum rückende Bilder treten und wenn (nachprüfbare oder auch nicht unmittelbar nachprüfbare) Lügen und Falschaussagen ungeachtet der über sie hereinbrechenden Entrüstung ${ }^{15}$ unbeirrt wiederholt und ausgebaut werden, dann sind das manipulative und im Effekt demagogische rhetorische Strategien, die ein zentrales Instrument für die politischen Zwecksetzungen Trumps sind. Eine Analyse der Sprache in Verbindung mit den damit vermittelten Argumenten oder anti-argumentativen Strategien ist daher unverzichtbar für eine

\footnotetext{
${ }^{15}$ Vgl. Kessler u. a. 2019, eine Liste aller oder doch sehr vieler falscher Aussagen von Trump mit Richtigstellungen bzw. der Ergänzung fehlender Gegenargumente.
} 
Aufklärung der Zusammenhänge und der durch diese Manipulationen verdeckten politischen Sachlagen.

Damit erweisen sich die Beleidigungen und narrativen Einbettungen dieser Invektiven ebenso wie die Dekontextualisierungen und Emotionalisierungen als Sonderfälle zweier allgemeinerer Strategien:

\section{Strategie: Das Vermeiden diskursiver Praktiken insgesamt}

Von Trump geäußerte Meinungen werden so gerahmt - durch die Wahl eines bestimmten Mediums, durch das Zitat von vermeintlichen oder wirklichen Expert*innen, mittels der Autorität seines Amtes und vor allem mit Verweis auf die Bosheit, Faulheit, Hintertriebenheit usw. der jeweiligen Gegner oder schlicht durch die schiere Wiederholung -, dass sie als zwingend erscheinen, ohne dass dafür eine Begründung geliefert würde.

\section{Strategie: Nebenschauplätze und formale Details werden als Argumente für generelle Schlussfolgerungen genutzt}

Es ist eine in allen Attacken auf Gegner und bei allen Selbstdarstellungen Trumps zu beobachtende Strategie, dass er die Diskussion nicht bei den harten Sachthemen sucht. Ein schlagendes Beispiel dafür ist die Verleihung des von Trump erfundenen „Fake News Award“ für falsche, unehrliche Berichterstattung durch die Medien, den Trump am 17. Januar 2018 in dem Blog der GOP-Website veröffentlichte. ${ }^{16}$ In der Liste der ,Gewinner' zeigte sich, dass gerade die Form einer solchen Auflistung Trumps Kommunikationsteam Probleme bereitete. Denn unter den Preisträger*innen befanden sich nahezu ausschließlich Meldungen über Nebensächliches wie z. B. einen Bericht, dass Trump angeblich bei seinem ersten Staatsbesuch in Japan während des Rahmenprogramms Fische falsch gefüttert haben soll. Nur ein Listeneintrag bezog sich direkt auf die Berichterstattung zu den Russland-Kontakten von Trumps Wahlkampfteam, also auf ein politisch relevantes und für Trump brisantes Thema, nämlich der elfte und letzte. Doch dieser präsentiert nur die Wiederholung einer pauschalen Kritik: „And last, but not least: RUSSIA COLLUSION! Russian collusion is perhaps the greatest hoax perpetrated on the American people. THERE IS NO COLLUSION!“" (Team GOP 2017) Weder die Meldungen über politisch Nebensächliches noch die Pauschalbehauptung können die Aussage belegen, die Mainstream-Presse sei aufgrund ihrer Falschberichterstattung der „Feind des amerikanischen Volkes“. Die Awards zeigen außerdem, dass auch Trumps Administration sich irgendwie doch noch an Fakten und Belegbarkeit gebunden fühlt, zumindest in diesem

\footnotetext{
${ }^{16}$ Vgl. Team GOP 2017. Die Seite war vom 17. Januar bis 6. Februar 2018 abrufbar und ist seit dem 7. Februar 2018 ebenso wie die gesamte Domain offline. Für eine Archiv-Kopie, vgl. Angabe im Literaturverzeichnis. Zitate gibt es in allen großen Zeitungen und Medienanstalten am 17. bzw. 18. Januar 2018.
} 
Medium einer zur Überprüfung auffordernden „Roten Liste“. Dadurch wird nun das Gegenteil sichtbar zu Trumps Intentionen, nämlich, dass der große Diskurs, die große Erzählung Trumps eben gerade nicht belegbar ist. ${ }^{17}$

Wie sehr Methode und politische Strategie zusammenhängen und wie unverzichtbar es ist, sich mit beidem zu beschäftigen und in sozialwissenschaftliche Untersuchungen als Komplement aufzunehmen, decken Texte auf, die aus einem ganz anderen zeitlichen und kulturellen Horizont stammen: nämlich Texte aus dem antiken 4. Jahrhundert v. Chr. in Griechenland. Exemplarisch möchte ich die Aufmerksamkeit auf die Dialoge des griechischen Philosophen Platon lenken. Auch wenn die Athener Demokratie, innerhalb deren sich Platons Texte und Protagonist*innen bewegen, große Unterschiede zu unserer heutigen repräsentativen Demokratie aufweist, und auch wenn die Kommunikationswege und -prozesse freilich - allein schon aufgrund der zahlenmäßig begrenzten Bürger*innenschaft Athens und des Fehlens von digitalen Massenmedien - andere gewesen sind, ein wichtiger Aspekt bleibt doch über diese Unterschiede hinweg im Grundsätzlichen gleich: nämlich der Unterschied zwischen verschiedenen Niveaus der Auseinandersetzung und zwischen dem Bestreben, sich dem argumentativen Diskurs zu entziehen oder aber diesen Diskurs gerade rational mit hinreichenden Begründungen zu versehen.

\section{Was braucht man für eine begründete und begründende Politik? Analysen aus der antiken Rhetoriktheorie}

Platon entwickelt seine Theorie der Rhetorik und Politik vor allem in den beiden großen Dialogen Gorgias und Phaidros. ${ }^{18}$ Doch auch eine Reihe weiterer Dialoge ist mit diesen und ihren Themen eng verwoben und bildet mit ihnen eine Art gedankliches Netz, in dem der eine Knotenpunkt auf den anderen weiterverweist. $\mathrm{Zu}$ diesen mit Gorgias und Phaidros verflochtenen Dialogen gehört auch der Protagoras, in dem vor allem Fragen der richtigen Methode und des zu bevorzugenden Mediums für eine politische und philosophische Auseinandersetzung diskutiert werden.

In diesem platonischen Dialog Protagoras trifft nun Sokrates auf den Sophisten Protagoras, der sich gerade als Gast des reichen Kallias, des Sohnes des Hipponikos, in Athen die Ehre gibt und verspricht, den jungen Leuten alles beizubringen, was sie für eine politische Karriere benötigen. Er verspricht, sie zu guten Bürgern und erfolgreichen politischen Funktionären zu machen. Sokrates begleitet den jungen Adeligen Hippokrates, Sohn des Apollodoros, der bei dem berühmten Lehrer Unterricht nehmen möchte, will aber zunächst prüfen, ob der diesem erstaunlichen Anspruch überhaupt genügen kann. Man ahnt schon: Sokrates

\footnotetext{
${ }^{17}$ In der Ankündigung des Awards heißt es: „2017 was a year of unrelenting bias, unfair news coverage, and even downright fake news.“ (Team GOP 2017; 17:00 - 17. Januar 2018, @ realDonaldTrump)

${ }^{18}$ Eine ausführliche Interpretation habe ich vorgelegt in Uhlmann 2019.
} 
hat berechtigte Zweifel, dass der Starlehrer, der von einer großen Schar von Bewunderern umgeben ist und sich von diesen feiern lässt, wirklich den jungen Leuten die „Aretè“, die Tugend des guten Bürgers, beizubringen in der Lage ist. Bei diesem Besuch zeigt sich nun sogleich, dass der Redner und Lehrer Protagoras es nicht kurz mag, sondern dass er ein Freund langer und die Worte wohl formender Reden ist. Wortreich beantwortet er Sokrates` Fragen und besonders die eine Frage, deretwegen dieser gekommen war: „Ist die Tugend denn überhaupt lehrbar, von der du behauptest, sie unterrichten zu können und für deren Unterricht du dein beträchtliches Honorar forderst?"19

Doch nachdem Protagoras als Antwort zunächst eine mythische Erzählung und dann etwas, was er Begründung und Beweise nennt (Prt. 323a), vorgetragen hat, behauptet Sokrates zwar, als er später von dieser Begegnung erzählt, er sei bezaubert gewesen und hätte sich gar nicht satt hören können an den Worten, er bekennt aber auch, dass ihn eine, wie er sagt, Kleinigkeit an dieser Rede gestört und ihm gefehlt habe (Prt. 328e, 329b). Von dieser Kleinigkeit stellt sich allerdings im Laufe des Dialogs heraus, dass dieses Problem die Richtigkeit der gesamten Äußerungen des Protagoras aufhebt oder infrage stellt. Um also diese „,kleine“ Frage auch noch zu klären, verwickelt Sokrates Protagoras in ein dialektisches Gespräch, für das er auch gleich die Regeln bestimmt: kurze Antworten, außerdem Hinhören und Aufnehmen, was der andere sagt.

Doch was hat Sokrates nun an der Rede des Protagoras eigentlich gestört? Es ist die mangelnde rationale Qualität der Rede. Sokrates zeigt nach und nach, dass Protagoras tatsächlich gar keine Beweise oder rationalen Argumente angeführt, sondern lediglich Geschichten aus dem Leben von häufig zu beobachtenden Verhaltensweisen erzählt hat. Auch der Kulturentstehungsmythos, mit dessen stilistischer und narrativer Brillanz Protagoras seine Zuhörer beeindruckt, ${ }^{20}$ hat als Ausgangspunkt und Substanz die gleichen alltäglichen Meinungen und Gewohnheiten, denn Protagoras erfindet die Geschichte davon, wie der Mensch bei der Vergabe der lebensrettenden Eigenschaften und Stärken an alle Lebewesen einfach vergessen wurde. Der Heros Prometheus habe als Ausgleich dafür die Weisheit der Göttin Athene, die Handwerkskunst des Hephaistos und das Feuer aus dem Olymp gestohlen (Prt. 321c-322a). Weil die Menschen aber noch keine Fähigkeiten für das Zusammenleben besessen hätten, hätten sie gedroht, sich durch Streit und Kriege selbst zu vernichten, weshalb ihnen Zeus durch den Götterboten Hermes zwei Gaben habe schicken lassen mit dem Auftrag, diese an alle gleichmäßig zu verleihen: nämlich Aidos und Dike, Scham und Recht (Prt. 322b-d).

\footnotetext{
${ }^{19}$ Platon charakterisiert die Sophisten an vielen Stellen seines Werks negativ durch die Betonung ihres kommerziellen Unterrichts, für den ein Honorar verlangt wurde, z. B. Ap. 19d-e, 31b-c, 33a-b. Dies ist für ihn ein Argument dafür, dass die Sophisten keine wahren Philosophen sein können: R. 485e. Vgl. Tell 2009; Blank 1985; vgl. eine gegenüber Platon sehr kritische Position bei Schriefl 2013.
}

${ }^{20} \mathrm{Vgl}$. zum Mythos den erkenntnisreichen Kommentar von Manuwald 1999, 170-179. 
Die beiden Teile des Mythos dienen Protagoras als Stoff, um erklären zu können, dass die Menschen zwar nicht alle dieselben Künste und Handwerke beherrschten, dass sie aber alle Anteil an den politischen Tugenden hätten. Die auf die eigentliche Mythos-Erzählung folgenden Begründungen wollen zusätzlich noch begründen, dass und warum wir alle die politische Tugend für lehrbar halten und dass es in der Erziehung darauf ankomme, den Kindern zuallererst diese Fähigkeiten zu vermitteln. Dies geschieht dadurch, dass Protagoras vor Augen führt, wie sich die Menschen zumeist verhielten, was sie lobten und was sie tadelten. Diese Geschichten haben den Zweck, zu beweisen, dass wir alle immer schon im Besitz einer gewissen Tugend sind und dass wir sie auch stetig anderen zu vermitteln bemüht sind. Anstelle von Beweisen und rationalen Argumenten bringt Protagoras dabei lediglich Indizien und Rückschlüsse aus unseren üblichen Meinungen und unserem Verhalten. Daher sind in Sokrates 'Augen auch die Beweise und Argumentationen, die Protagoras in seiner langen Rede vorgeführt hat, keineswegs hinreichende Beweise, wie Protagoras behauptet (Prt. 323a und c8-d1), sondern nichts anderes als Geschichten, Erzählungen, die nicht auf der begrifflichen Ebene stattfinden und dementsprechend auch keine beweisende Qualität besitzen.

Sokrates vergleicht Protagoras mit „einem von unseren Volksrednern“ (demegoron, Prt. 329a1). ${ }^{21}$ Diese nämlich könnten zwar gut Reden halten, aber wenn jemand dazu näher nachfragte, dann seien sie wie die Bücher. Sie könnten keine weitere Auskunft geben. ${ }^{22}$ Und wenn sie antworteten, dann würden sie gleich in eine übermäßig lange Rede fallen. Dies sei so, wie wenn Metall, das einmal angestoßen wurde, lange weitertönt (Prt. 329a5-b2). Protagoras hingegen könne sowohl lang als auch kurz sprechen. Das ist freilich sokratische Ironie, denn Sokrates antizipiert bereits, dass Protagoras die entscheidenden Begründungsfragen nach der Lehrbarkeit der Tugend und danach, was die Tugend überhaupt ist (vgl. Prt. 360e-361a und ähnlich Men. 71a.), nicht wird beantworten können und dass er sich aus diesem Grund gegen die Form des dialektischen Gesprächs wehrt, das ihn dazu zwingen könnte, begrifflich zu argumentieren. Für Platon ist das dialektische Gespräch hingegen diejenige Form der Kommunikation, in der am besten Argumente gegeneinander abgewogen, Widersprüche aufgedeckt und hinreichende begriffliche Begründungen gemeinsam ermittelt werden können.

In diesem Gespräch über die Hauptfrage des Dialogs - Ist die Tugend lehrbar? - wird nun die von Protagoras zu Beginn wortreich und ästhetisch und stilistisch ansprechend formulierte These in allen ihren Teilen widerlegt. Die Folge ist, dass Protagoras sich aus dem Gespräch zurückziehen möchte. Ganze 14 Seiten (in der Stephanuspaginierung: Prt. 334c-348c) benötigt es, bis das eigentliche Gespräch fortgesetzt werden kann. Das zeigt nicht nur die Widerständigkeit des Protagoras und seine Strategien, mit denen er versucht, sich einer sachlichen

\footnotetext{
${ }^{21}$ An anderer Stelle habe ich die Zusammenhänge zwischen dem griechischen Begriff ,demegoria“ oder ,demegorein “ und unserem Wort ,Demagogie“ dargestellt; vgl. Uhlmann 2019; zur Bestimmung Trumps als Demagoge vgl. auch Mercieca 2015.

${ }^{22}$ Ein ähnliches Argument findet sich in der berühmten Schriftkritik im Phaidros: Phdr. 275d-e.
} 
Diskussion zu entziehen und damit der Widerlegung zu entgehen, sondern auch, welche Bedeutung Platon der Methode der Erkenntnisarbeit beimisst. Es kommt eben doch darauf an, das richtige Medium und die richtigen Methoden zu suchen, um zu einer sicheren Erkenntnis zu kommen. Bereits die Methode kann ein wichtiges Indiz für die Qualität der vertretenen Meinung sein. Das kann man daran erkennen, dass Platon die anderen anwesenden Sophisten - Hippias und Prodikos - beide nur von der Länge oder Kürze der Rede sprechen lässt, so als wäre die Anzahl der verwendeten Worte das entscheidende Kriterium für die Qualität der Rede. Sokrates hingegen sagt nur, er wolle, dass die Rede dem Sachverhalt angemessen sei. Tatsächlich hält auch Sokrates selbst durchaus längere Reden, allerdings solche, die sich nicht um eine Begründung drücken, sondern diese im Gegenteil suchen und herausfordern.

Unter den Anwesenden ist auch der junge Alkibiades, der spätere Feldherr und Initiator der für Athen desaströs endenden Sizilienexpedition. Dieser war in jungen Jahren von Sokrates und seiner Philosophie fasziniert und spricht daher auch für dessen Seite. Er sagt, wenn Protagoras weiterhin behaupten wolle, er könne ebenso gut kurz wie lang sprechen, dann

„solle er nicht bei jeder Frage eine lange Rede ausbreiten, den Argumenten ausweichen und anstatt dem anderen Rechenschaft zu geben, die Rede so in die Länge ziehen, solange bis die meisten der Zuhörer vergessen haben, worüber die Frage eigentlich ging" (Prt. $336 c-d)$.

Damit spricht Alkibiades eine antidiskursive Ausweichstrategie an und benennt damit zugleich zwei Praktiken, die ganz generell einander entgegengesetzt sind und die in den platonischen Dialogen durch Sokrates auf der einen Seite und die Sophisten auf der anderen Seite repräsentiert werden: Entweder nämlich gibt man dem anderen Rechenschaft über die eigene Meinung oder man weicht den Argumenten aus, um nicht in die Gefahr zu geraten, argumentieren zu müssen oder widerlegt zu werden.

\section{Methode und Politik}

Wir haben bei Donald Trump in unserem Kurzüberblick bereits gesehen, dass seine Kommunikationsstrategien auf die eben exemplifizierten Formen des Antidiskursiven abzielen. Es gibt aber noch eine Gemeinsamkeit in den Analysen. Denn auch über die richtige Etikette bei der Diskussionsrunde wird im Protagoras debattiert. Nachdem sich Protagoras zunächst wie gesehen aus dem Gespräch zurückgezogen hat, appellieren die anwesenden Zuhörer an die Diskutanten, dass man sich im gegenseitigen Wohlwollen streiten, aber nicht feindselig attackieren solle (Prt. 337a-e). Zu solchen Angriffen war es tatsächlich (noch) nicht gekommen, die Gefahr liegt aber offen zutage: Die Gesprächspartner sollten nichts gegen ihre Überzeugung sagen, nur um durch Überredung zu beeindrucken, sondern bei der Sachauseinandersetzung bleiben (Prt. 337a-337c). 
Die Gesprächspartner Sokrates und Protagoras werden auch im Folgenden diese Grenze nicht überschreiten. Der Grund dafür ist bei Protagoras, dass er sich an den allgemeinen gesellschaftlichen Konsens über tugendhaftes und untugendhaftes Verhalten gebunden fühlt - denn das ist das Material, mit dem er als Lehrer arbeitet und gesellschaftlich anschlussfähig bleibt. Man kann in anderen Dialogen besonders im Dialog Gorgias - erkennen, dass dieses Tabu gebrochen werden kann, wenn ein Dialogpartner wie z. B. Kallikles seinen eigenen materiellen und gesellschaftlichen Erfolg absolut setzt und erwartet, auch mit dieser These vom Recht des Stärkeren akzeptiert zu werden.

Es ist heute viel darüber geschrieben worden, wie es sein kann, dass Trump derart offen lügt, ${ }^{23}$ seine Gegner attackiert und beleidigt, dass er diplomatische Gepflogenheiten und Etikette auf dem Parkett der internationalen Politik ganz und gar missachtet und für sich selbst neu definiert. Der Grund scheint mir aus rhetorikanalytischer Sicht zu sein, dass seine Rhetorik selbst eine neue, alternative Form politisch akzeptablen Verhaltens definiert, mit dem er durch die Abgrenzung von anderen Gruppen ein Überlegenheits- und ein Identitätsbewusstsein aufbaut, für welches auch harsche Töne gegenüber dem uneinsichtigen politischen Gegner nicht nur erlaubt, sondern sogar zuträglich sind.

$\mathrm{Zu}$ diesen neuen Formen der Kommunikation gehört es auch, dass er es als akzeptabel definiert, politisch zu agieren, ohne rationale Argumente vorzubringen oder sich mit denen anderer auseinanderzusetzen. Unter die politischen Praktiken, die Trump gemeinsam mit seinem Kommunikationsteam definiert, müssen wir auch die Neudefinition von Diskurs und Diskursverknüpfungen über die Kurznachrichtenplattform Twitter zählen. Wie wo mit wem geredet oder zu wem gesprochen wird, bestimmt Trump selbst und wirft dabei traditionelle Gepflogenheiten bewusst über Bord. So erscheint es - offenbar - mittlerweile als akzeptabel, dass Trump die Medien nur noch pauschal verurteilt und regelmäßig über unfaire Berichterstattung klagt, ohne zu der Kritik oder den Berichten sachlich ausführlich Stellung zu nehmen. Eine detaillierte Analyse wird durch Trumps Twitter-Stil sogar als ,beckmesserisch“ oder verlogen diskreditiert. Denn Faktenchecks und das Auseinandernehmen von Äußerungen Trumps missachte die - in Trumps Darstellung - großen Erfolge seiner Präsidentschaft, die großen Linien und Aufgaben, und erhebe sich nach Art kleinlicher Intellektueller über die von Trump repräsentierte Mehrheit. ${ }^{24}$

\footnotetext{
${ }^{23}$ Der Band Skinnell 2018a ist diesem Thema in größeren Teilen gewidmet.

${ }^{24} \mathrm{Z}$. B. ,The White House is running very smoothly and the results for our Nation are obviously very good. We are the envy of the world. But anytime I even think about making changes, the FAKE NEWS MEDIA goes crazy, always seeking to make us look as bad as possible! Very dishonest.“ (03:59 - 15. November 2018, @ realDonaldTrump); „The Trump Administration has accomplished more than any other U.S. Administration in its first two (not even) years of existence, $\&$ we are having a great time doing it! All of this despite the Fake News Media, which has gone totally out of its mind - truly the Enemy of the People!“ (14:43 - 9. Dezember 2018, @ realDonaldTrump); „With all of the success that our Country is having, including the just released jobs numbers which are off the charts, the Fake News \& totally dishonest Media concerning me and my presidency has never been worse. Many have become crazed lunatics who have given up on the TRUTH! [...].“(04:56 - 7. Januar 2019, @ realDonaldTrump)
} 


\section{Schlussfolgerungen}

Trumps Twitter-Strategien sind eben deshalb rhetorisch und demagogisch manipulativ, weil sie sich mittels des Kurznachrichtenmediums sowie auch durch die Veränderung der Spielregeln der politischen Kommunikation und Aufhebung diskursiver Grenzen einem intellektuellen rationalen Diskurs entziehen und stattdessen Bilder - und zwar sprachlich entworfene ebenso wie solche im Bildmedium - erschaffen, die alle Diskursteilnehmer, die sich darauf einlassen, von einer mühsamen, gründlichen Auseinandersetzung mit der aktuellen politischen Lage und der Vielheit unterschiedlicher politischer Meinungen freisprechen und die leicht zu rezipieren und memorieren sind. Die Aussage, die diese neuen Formen der Kommunikation als Tenor begleitet, lautet: ,Alle diejenigen, die mich kritisieren, gehören einer abgehobenen Elite an, die die Sorgen und Wünsche der Mehrheit der Menschen nicht kennt und für sie auch kein offenes Ohr hat, sondern sich in einen Privatdiskurs der Intellektuellen zurückzieht. $\mathrm{Zu}$ diesem und nur zu diesem gehöre es, die Fakten zu prüfen und Argumente gegeneinander abzuwägen. ' Deren Ziel sei dabei immer die Abwertung Trumps und mit ihm der hart arbeitenden Mehrheit der Amerikaner. Trump greift damit eine lange in den USA etablierte Elitenkritik und Kritik an ,denen da in Washington “ auf und treibt sie durch seine neue antirationale Kommunikationspraxis auf die Spitze.

Für die Zwecke dieser Strategien ist Twitter das ideale Medium. Es ist eine Plattform, in der der Nutzer Trump die Diskurse bunt miteinander vermischt und daraus ein einheitlich erscheinendes Narrativ von seiner Person und seinen Leistungen als Präsident erschafft. Falschaussagen, Beleidigungen, das Missachten der Etikette der internationalen Politik, das Überhören oder bloße Ablehnen und Verhöhnen anderer politischer Meinungen und auch das Aufkündigen eines kontinuierlichen Informationsdialogs zwischen Weißem Haus und den dort akkreditierten Journalist*innen sind Instrumente und Effekte dieses Narrativs, die nicht verdeckt, sondern im Gegenteil durch das Reflektieren darüber und Erinnern daran selbstbewusst als neue Form der Politik präsentiert werden. Wenn Trump die journalistischen Fragen und das Zusammentreffen mit den Journalist*innen in seinen politischen Alltag, in seine Sitzungen und Besuche ausländischer Gäste integriert, ihnen daher aber auch nur noch die Stellung von Zuschauer*innen oder eine Nebenrolle zugesteht, dann wird die traditionelle Kommunikationskultur zwischen Politik und Presse in etwas umdefiniert, das Trump mit seiner Twitter-Kurz-Strategie vollständig beherrschen und bestimmen kann. Das demokratische Gleichgewicht sowie auch der gesellschaftliche Zusammenhalt können dadurch in eine Schieflage geraten.

Wie wir am Beispiel des platonischen Dialogs Protagoras gesehen haben, ${ }^{25}$ kann eine Anleitung zur Analyse solcher Praktiken und der Verflechtungen von Kommunikationsmedien, rhetorischen Strategien und politischer Agenda einen

\footnotetext{
${ }^{25}$ Für ausführliche Analysen zu weiteren für diesen Kontext relevanten rhetoriktheoretischen Texten von der Antike bis in die Gegenwart vgl. Uhlmann 2019.
} 
Beitrag dazu leisten, solche Schieflagen zu adressieren. Der Gedanke, den Platon formuliert, ist auch für uns heute noch bedenkenswert: Politische Rhetorik kann mit der Wahl ihres Mediums und ihrer Methoden einen Mangel an rationaler Begründung und eine Schwäche der politischen Positionen des Redners oder der Rednerin zu verdecken versuchen. Es ist die Aufgabe von Rhetoriker*innen ebenso wie die aller mündigen Bürgerinnen und Bürger, solche verborgenen Manipulationen aufzudecken und dazu beizutragen, die politische Kommunikation auf das Niveau einer differenzierten, verschiedene Argumente abwägenden rationalen Diskussion zurückzuführen.

\section{Literatur}

Blank, David L.: Socratics versus Sophists on payment for teaching. In: Classical Antiquity 4/1 (1985): 1-49.

Brady, William J./Wills, Julian A./Jost, John T./Tucker, Joshua A./Bavel, Jay J. Van: Emotion shapes the diffusion of moralized content in social networks. In: Proceedings of the National Academy of Sciences of the United States of America 114/28 (2017): 7313-7318.

Breuninger, Kevin: White House Press Briefings have all but vanished - and Trump's control of the message is stronger than ever (03.10.2018), https://www.cnbc.com/2018/10/02/whitehouse-press-briefings-vanish-trump-takes-more-control.html (30.01.2018).

Davis, William P.: ,Enemy of the People': Trump breaks out this phrase during moment of peak criticism. In: The New York Times (20.07.2018), https://www.nytimes.com/2018/07/19/business/media/trump-media-enemy-of-the-people.html (31.01.2019).

Franke-Ruta, Garance: Is Elizabeth Warren native american or what? In: The Atlantic (20.05.2012), https://www.theatlantic.com/politics/archive/2012/05/is-elizabeth-warren-native-american-or-what/257415/ (31.01.2019).

Guterman, Jeffrey: Alternative facts: Kellyanne interview by Chuck Todd. In: NBC News (22.01.2017), https://www.youtube.com/watch?v=Y7np8BDNd7Y (30.01.2019).

Haberman, Maggie/Oppel, Richard A. Jr.: Donald Trump criticizes muslim family of slain U.S. Soldier, Drawing Ire. In: The New York Times (30.07.2016), https://www.nytimes. com/2016/07/31/us/politics/donald-trump-khizr-khan-wife-ghazala.html (31.01.2019).

Kessler, Glen/Kelly, Meg/Rizzo, Salvador/Ye Hee Lee, Michelle: In 730 Days, President Trump has made 8,158 false or misleading claims. In: The Washington Post (19.05.2017), https:// www.washingtonpost.com/graphics/politics/trump-claims-database/?noredirect=on\&utm term $=.094 \mathrm{e} 27 \mathrm{c} 3 \mathrm{daf} 5$ (30.01.2019).

Manuwald, Bernd: Platon: Protagoras. Übersetzung und Kommentar. Göttingen 1999.

McArdle, Mairead: Sarah Huckabee Sanders defends suspension of WH Press Briefings. In: The National Review (23.01.2019), https://www.nationalreview.com/news/sarah-huckabee-sanders-defends-suspension-of-wh-press-briefings/ (30.01.2019).

Mercieca, Jennifer: The Rhetorical brilliance of Trump the demagogue. In: The Conversation (11.12.2015), https://theconversation.com/the-rhetorical-brilliance-of-trump-the-demagogue-51984 (30.01.2019).

Müller, Jan-Werner: Was ist Populismus? Ein Essay. Berlin 2016.

Platon: Platonis opera, tomus I, hg. von E. A. Duke/ W. F. Hicken/W.S.M. Nicoll/D. B. Robinson/J. C. G. Strachan, Oxford 1995 (=Ap.).

Platon: Platonis opera. 5 Bde., hg. von John Burnet. Oxford 1900-1907 (= Prt./R.).

Samuels, Brett: White House Press briefings few and far between during summer months. In: The Hill (31.08.2018a), https://thehill.com/homenews/administration/404533-as-august-comes-toa-close-fewer-press-briefings-becomes-the-norm (30.01.2019). 
Samuels, Brett: Sanders defends lack of White House Press briefings. In: The Hill (30.09.2018b), https://thehill.com/homenews/administration/409155-huckabee-sanders-defends-lack-of-press-briefings (30.01.2019).

Schriefl, Anna: Platons Kritik an Geld und Reichtum. Berlin/Boston 2013.

Skinnell, Ryan (Hg.): Faking the News. What Rhetoric Can Teach Us About Donald J. Trump. La Vergne 2018a.

Skinnell, Ryan: What passes for truth in the Trump era. Telling it like it isn't. In: Ders. (Hg.): Faking the News. What Rhetoric Can Teach Us About Donald J. Trump. La Vergne 2018b: 76-94.

Smith, Allan: Trump on Obamacara's rising popularity: ,There's nothing to love - It's a disaster, folks, OK?‘ In: Business Insider (27.02.2017), http://www.businessinsider.de/trump-obamacares-approval-ratings-2017-2? $\mathrm{r}=\mathrm{US} \& \mathrm{IR}=\mathrm{T}$ (30.01.2019).

Stöber, Rudolf: Ohne Redundanz keine Anschlusskommunikation. Zum Verhältnis von Information und Kommunikation. In: Medien \& Kommunikationswissenschaft 59/3 (2011): 307-323.

Team GOP: The highly-anticipated 2017 fake news awards, https://web.archive.org/ web/20180118120302/gop.com/the-highly-anticipated-2017-fake-news-awards/ (18.01.2017).

Tell, Håkan: Wisdom for sale? The Sophists and money. In: Classical Philology 104/1 (2009): $13-33$.

Uhlmann, Gyburg: Rhetorik und Wahrheit. Ein prekäres Verhältnis von Sokratis bis Trump. Berlin 2019.

Urback, Robyn: Take it from the Republican faithful - Trumps's not a liar, just an idiot. In: $C B N$ News (08.06.2017), https://www.cbc.ca/news/opinion/trump-comey-testimony-1.4152589 (11.02.2019).

Washington Times Staff: Trump vs. CNN's Jim Acosta: The full exchange. In: The Washington Times (07.11.2018), https://www.washingtontimes.com/news/2018/nov/7/trump-vs-cnns-jimacosta/ (30.01.2019).

Winck, Ben/Skankar, Vivek: White House Press Briefings are getting shorter. In: Bloomberg (17.08.2018), https://www.bloomberg.com/news/articles/2018-08-17/ask-me-no-questionswhite-house-briefings-get-shorter-chart (30.01.2018).

Open Access Dieses Kapitel wird unter der Creative Commons Namensnennung 4.0 International Lizenz (http://creativecommons.org/licenses/by/4.0/deed.de) veröffentlicht, welche die Nutzung, Vervielfältigung, Bearbeitung, Verbreitung und Wiedergabe in jeglichem Medium und Format erlaubt, sofern Sie den/die ursprünglichen Autor(en) und die Quelle ordnungsgemäß nennen, einen Link zur Creative Commons Lizenz beifügen und angeben, ob Änderungen vorgenommen wurden.

Die in diesem Kapitel enthaltenen Bilder und sonstiges Drittmaterial unterliegen ebenfalls der genannten Creative Commons Lizenz, sofern sich aus der Abbildungslegende nichts anderes ergibt. Sofern das betreffende Material nicht unter der genannten Creative Commons Lizenz steht und die betreffende Handlung nicht nach gesetzlichen Vorschriften erlaubt ist, ist für die oben aufgeführten Weiterverwendungen des Materials die Einwilligung des jeweiligen Rechteinhabers einzuholen. 